\section{Actividad antibacteriana in vitro del extracto etanólico de propóleo peruano sobre Streptococcus mutans y Lactobacillus casei}

In vitro antibacterial activity of peruvian propolis' ethanolic extract on Streptococcus mutans and Lactobacillus casei

\section{Resumen}

Con el objeto de determinar la acción antibacteriana del extracto etanólico del propóleo peruano (EEPP) proveniente del Valle de Oxapampa (Pasco); mediante el método de difusión en Placa se usó las cepas Streptococcus mutans ATCC 25175 y Lactobacillus casei ATCC 393, para enfrentarlas a las soluciones: 0,8, 20 y $30 \%$ v/v del EEPP, y compararlas a los testigos Clorhexidina 0,12 \% y alcohol 70 \%. Se determinó que la acción antibacteriana del EEPP contra $S$. mutans muestra una mayor tendencia de actividad inversamente proporcional a su concentración, que en el caso del L. casei; tal acción antibacteriana en las concentraciones $0,8,20$ y 30 \% es significativa en comparación al testigo negativo; así mismo la acción contra $S$. mutans es mayor que en $L$. casei; siendo significativas en las concentraciones de 0,8 y $20 \%$; y también la acción antibacteriana del EEPP al 0,8 \% es mayor que la acción de la Clorhexidina, tanto para S. mutans y L. casei. Se concluye que EEPP en solución al 0,8 \% tiene una mejor acción antibacteriana contra $S$. mutans y $L$. casei que la Clorhexidina al 0,12\%.

\section{Abstract}

With the intention of determining the antibacterial action of the ethanolic extract of Peruvian propolis (EEPP) originally from Oxapampa's Valley (Pasco); using the method of diffusion in Plate the vine-stocks Streptococcus mutans ATCC 25175 and Lactobacillus casei ATCC 393 were used, and faced to the solutions: 0,8, 20 and $30 \% \mathrm{v} / \mathrm{v}$ of the EEPP, and compared them to the witnesses Clorhexidine $0,12 \%$ and alcohol $70 \%$. It was determined that the antibacterial action of the EEPP against $S$. mutans shows a greater tendency of inversely proportional activity in its concentration, to that of L. casei case; such antibacterial action in concentrations $0,8,20$ and $30 \%$ is significant in comparison to the negative witness; also the action against $S$. mutans is greater than in L. casei; being significant in the concentrations of 0,8 and $20 \%$; and also the antibacterial action of the EEPP to $0,8 \%$ is greater than the action of Clorhexidine, as much for S. mutans and L. casei. One concludes that EEPP in solution to $0,8 \%$ has a better antibacterial action against $S$. mutans and L. casei that the Clorhexidine to $0,12 \%$.

\section{Marly Eguizábal A', Hilda Moromi Nakata $^{2 *}$}

Bachiller en Odontología. 2Departamento Académico de Ciencias Básicas, Laboratorio de Microbiología.

1,2 Facultad de Odontología de la Universidad Nacional Mayor de San Marcos. Lima Perú.

Correspondencia:

Marly Eguizabal A.

Bachiller en Odontologia

Facultad Odontología, UNMSM

Av. Amezaga s/n, Lima, 1 Perú. Tlf: 6197000

e-mail: maea1982@hotmail.com

Palabras clave: Propóleo, actividad antibacteriana, Streptococcus mutans, Lactobacillus casei, Clorhexidina.

Key words: Propolis, antibacterial activity, Streptococcus mutans, Lactobacillus casei, Clorhexidine.

\section{Introducción}

La caries dental es una enfermedad multifactorial, de alta incidencia en el Perú, según el Ministerio de Salud, 98 de cada 100 peruanos presenta lesiones cariosas; ${ }^{1}$ a pesar de la fluorización de la sal de cocina, y la promoción de la eliminación de placa bacteriana por medios mecánicos como el cepillado dental. Lamentablemente, algunas personas no acceden a este tipo de sal y otros no regularizan el control de su higiene bucal. $^{2}$
Por tal motivo, en la actualidad existen los agentes antimicrobianos, llamados colutorios, que inhiben químicamente la formación o proliferación de la placa. Entre estos está la clorhexidina al $0,12 \%$, como el agente más utilizado en el medio dada su eficacia en la eliminación de microorganismos cariogénicos; desafortunadamente presenta efectos secundarios adversos, como disgeusia y tinción dental, razones que limitan su utilización. Cabe resaltar entonces que el hallazgo del agente antiplaca ideal aún constituye un reto para los investigadores.
De esa búsqueda, desde hace años viene creciendo la corriente que propone la utilización de productos naturales como solución a los problemas médicos y odontológicos. La medicina alternativa presenta a la Odontología, entre otros, al Propóleo como solución a diversos problemas de salud oral. De ahí que existe una gama de estudios sobre Propóleo de origen extranjero que comprueban su acción antibacteriana en el laboratorio contra bacterias Grampositivas: ${ }^{3} S$. salivarius, S. sanguis, S. mitis y Candida albicans. ${ }^{4,5}$ Otros autores han estudiado 
fracciones y componentes del Propóleo demostrando que la fracción $\mathrm{H}-\mathrm{fr}$ $\mathrm{y}$ los flavones (apigenin y $\mathrm{tt}$-farnesol) presentan alta actividad antibacteriana y poder inhibitorio de la Glucosil transferasa (GTF) con reducción de caries dental. ${ }^{6,7}$ Por otro lado en estudios clínicos se ha comprobado que un colutorio a base de Propóleo no presentó actividad antiplaca significativa, pero su actividad antibacteriana fue semejante a la clorhexidina, ${ }^{8}$ asimismo una crema dental con Propóleo redujo significativamente el promedio de piezas afectadas con caries dental; otro estudio determinó que el Propóleo tiene alta concentración de calcio $\left(\mathrm{Ca}^{2+}\right)$ y la dureza del esmalte mostró un aumento constante en proporción directa al aumento de concentración de la solución. ${ }^{10}$

La geografía peruana y su diversa vegetación se prestan para el desarrollo de la apicultura, al punto que está en vías de ser una industria. Actualmente la producción de Propóleo aún se realiza a pequeña escala, a través de su principal derivado: la tintura de Propóleo (solución alcohólica de concentración desconocida). Teniendo en cuenta que la calidad del Propóleo va- ría de acuerdo a la zona de producción, es importante resaltar que en el 2004 se ha estandarizado el Propóleo del valle de Oxapampa, departamento de Pasco, denominándolo Propóleo Peruano, comprobando que su producción es de alta calidad y, cuya dosis de toxicidad aguda es $>10,57 \mathrm{~g} / \mathrm{kg}$ de peso, con una sensibilidad cutánea a la exposición directa de $32 \mathrm{mg}$ de muestra. ${ }^{11}$

En tal sentido el objetivo de la investigación es determinar la acción antibacteriana del extracto etanólico de propóleo peruano contra cepas de $S$. mutans y L. casei, teniendo a la clorhexidina al 0,12\% como referente testigo positivo.

\section{Materiales y Método}

Mediante el método de difusión en placa, usando las cepas patrón liofilizadas de S. mutans ATCC 25175 y L. casei ATCC 393; se les sometió a la acción antibacteriana del Extracto etanólico del propóleo peruano (EEPP), procedente del valle de Oxapampa (Pasco), estandarizado siguiendo las técnicas de López del Villar ${ }^{11}$ y del Foro $^{12}$ en las soluciones de 0,8, 20 y $30 \% \mathrm{v} / \mathrm{v}$.
Para los efectos de contar con referentes, se usó a la clorhexidina al 0,12 \%, como testigo positivo; y al Alcohol 70 $\%$ como testigo negativo.

La disposición de los antibacterianos en la placa petri se muestra en la Fig 1.

Para el análisis estadístico de los resultados se aplicó la Prueba Levene y la Prueba " $\mathrm{t}$ " student.

\section{Resultados y Discusión}

En el Cuadro 1 se muestra la actividad antibacteriana del EEPP, en comparación a los testigos positivo y negativo. Se puede apreciar que la acción antibacteriana del EEPP contra $S$. mutans muestra una mayor tendencia de actividad inversamente proporcional a su concentración, que en el caso del L. casei; tal acción antibacteriana en las concentraciones $0,8,20$ y $30 \%$ es significativa en comparación al testigo negativo; asimismo, la acción contra $S$. mutans es mayor que en L. casei; siendo significativas en las concentraciones de 0,8 y $20 \%$; y también la acción antibacteriana del EEPP al 0,8\% es mayor que la acción de la clorhexidina, tanto para S. mutans y L. casei.

Cuadro 1. Promedios $(\mathrm{n}=10)$ de los halos de inhibición antibacteriana en $\mathbf{m m}^{*}$

\begin{tabular}{|c|c|c|c|c|c|c|c|c|c|}
\hline \multicolumn{5}{|c|}{ Streptococcus mutans } & \multicolumn{5}{|c|}{ Lactobacillus casei } \\
\hline \multicolumn{3}{|c|}{ Extracto etanólico del propóleo } & \multirow{2}{*}{$\begin{array}{c}\text { Clorhexi- } \\
\text { dina } \\
0,12 \% \\
\text { (Testigo } \\
\text { positivo) }\end{array}$} & \multirow{2}{*}{$\begin{array}{c}\text { Alcohol } \\
70 \% \\
\text { (Testigo } \\
\text { negativo) }\end{array}$} & \multicolumn{3}{|c|}{$\begin{array}{l}\text { Extracto etanólico del } \\
\text { propóleo }\end{array}$} & \multirow{2}{*}{$\begin{array}{c}\text { Clorhexi- } \\
\text { dina } 0,12 \\
\% \\
\text { (Testigo } \\
\text { positivo) }\end{array}$} & \multirow{2}{*}{$\begin{array}{c}\text { Alcohol } \\
70 \% \\
\text { (Testigo } \\
\text { negativo) }\end{array}$} \\
\hline $0,8 \%$ & $20 \%$ & $30 \%$ & & & $0,8 \%$ & $20 \%$ & $30 \%$ & & \\
\hline $\begin{array}{c}13,56 \text { acf } \\
\pm 3,04\end{array}$ & $\begin{array}{c}12,00 \text { acg } \\
\pm 1,87\end{array}$ & $\begin{array}{l}12,33 \mathrm{aeg} \\
\pm 1,75\end{array}$ & $\begin{array}{l}11,72 \mathrm{~g} \\
\pm 1,50\end{array}$ & $\begin{array}{l}9,61 \text { be } \\
\pm 1,98\end{array}$ & $\begin{array}{l}11,25 \text { acf } \\
\pm 1,60\end{array}$ & $\begin{array}{c}10,10 \text { acg } \\
\pm 1,30\end{array}$ & $\begin{array}{l}11,20 \text { aeg } \\
\pm 1,16\end{array}$ & $\begin{array}{l}10,26^{\mathrm{g}} \\
\pm 0,88\end{array}$ & $\begin{array}{l}8,90^{\text {be }} \\
\pm 1,54\end{array}$ \\
\hline
\end{tabular}

*Hay diferencia estadística significativa $(P<0,05)$ cuando las letrillas son diferentes: EEPP vs Testigo negativo; EEPP vs Clorhexidina Testigo positivo.

Estos hallazgos aún no pueden compararse con estudios similares, dado que los conocimientos en específico aún no están disponibles. Si embargo se conoce que el propóleo cuenta con flavones como apigenin, tt-farnesol, compuestos que presentan acción antibacteriana y anticaries. ${ }^{7}$ En el país sólo existe el análisis de propiedades antimicrobianas realizado dentro del estudio de estandarización; ${ }^{11}$ el cual comprobó la actividad antibacteriana contra Staphylococcus aureus, C. albicans $\mathrm{y}$ Asperguillus níger, por lo que esta investigación representa el primer paso para presentar los efectos antibacterianos en la cavidad oral.
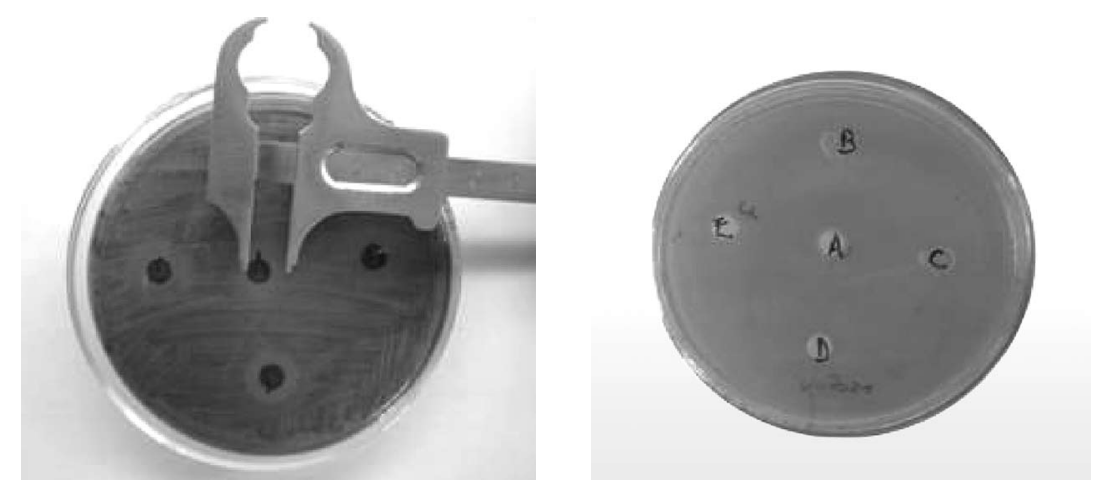

Fig. 1: Método de Difusión por Discos (Imagen de la izquierda: distribución de los discos; Imagen de la derecha: halo de acción antibacteriana): $A=E E P P ~ 0,8 \% ; B=E E P P 20 \% ; C=E E P P 30 \% ; D=$ Clorhexidina 0,12\% (Testigo positivo); $E=$ Alcohol $70 \%$ (Testigo negativo) 


\section{Conclusiones}

1. El extracto etanólico de propóleo peruano en solución al 0,8 \% tiene una mejor acción antibacteriana contra S. mutans y L. casei que la Clorhexidina al 0,12 \%.

2. La acción antibacteriana del extracto etanólico de propóleo peruano contra $S$. mutans muestra una mayor tendencia de actividad inversamente proporcional a su concentración, que en el caso del L. casei.

3. La acción antibacteriana del extracto etanólico de propóleo peruano en las concentraciones $0,8,20$ y $30 \%$ es significativa en comparación al testigo negativo.

4. La acción antibacteriana del extracto etanólico de propóleo peruano contra $S$. mutans es mayor que en L. casei; siendo significativas en las concentraciones de 0,8 y $20 \%$.

5. La acción antibacteriana del extracto etanólico de propóleo peruano al $0,8 \%$ es mayor que la acción de la Clorhexidina, tanto para $S$. mutans y L. casei.

\section{Referencias bibliográfica}

1. Ministerio de Salud del Perú - Plan de Salud Bucal 2005 http://www.minsa. gob.pe/portal/campanas/sbucal/ Archivos/RM538-2005\% 20 plan $\% 20$ de $\% 20$ salud \%Bucal.pdf

2. Woodall IR, Dafoe BR. Tratado de Higiene Dental. 3ra Edición. Barcelona: Salvat Editores. 1991:419-422.
3. Castagna A, Pinto A, Manzini N, Matiuzzi M, Sae M, Ribeiro L. Actividad antimicrobiana in vitro de extracto alcohólico de própolis. Ciênc Rural 2004;34(1): 159-163

4. Bruschi ML, Lara EHG, Martins CHG, Vinholis AHC, Casemiro LA, Panzeri H, Gremião MPD. Preparation and Antimicrobial Activity of Gelatin Microparticles Containing Propolis Against Oral Pathogens. Taylor \& Francis 2006;32(2):229- 238.

5. Martins R, Pereira E, Lima Jr. S, Senna M, Mesquita R, Santos V. Effect of commercial etanol propolis extra with the in vitro growth of Candida albicans collected from HIV-seropositive and HIV-seronegative Brazilian patients with oral candidiasis. J Oral Sci 2002;44(1):41-48.

6. Hayacibara MF, Koo H, Rosalen PL, Duarte S, Franco EM, Bowen WH, Ikegaki M, Cury JA. In vitro and in vivo effects of isolated fractions of Brazilian propolis on caries development. J Ethnopharmacol 2005;101(1-3):110115.

7. Koo H, Pearson SK, Scott-Anne K, Abranches J, Cury JA, Rosalen PL, Park K, Marquis RE, Bowen WH. Effects of apigenin and tt-farnesol on glucosyltransferase activity, biofilm viability and caries development in rats. Oral Microbiol and Immunol 2003;17:337-343.

8. Dantas de Almeida RV, Dias de Castro R, Vieira Pereira MS, Queiroz de Paulo M, Pereira Santos J, Nascimento Padilha WW. Efeito Clínico de solução antiséptica a base de Própolis em crianzas cárie activas. Pesq Bras Odontoped Clin Integr, João Pessoa. 2006;6(1):87-92.
9. Gispert AE, Cantillo EE, Rivero LA, Padrón IM. Actividad anticaries de una crema dental con propóleos. Rev Cubana de Estomatol 2000;37(3):166170 .

10. Giamalia I, Steinberg D, Grobler S, Gedalia I. The effect of propolis exposure on microhardness of human enamel in vitro. J Oral Rehabil 1999;26:941-943

11. López del Villar J, Ubillús CM. Estandarización del propóleos del Valle de Oxapampa, Departamento de Pasco (Perú) como materia prima para su utilización a nivel industrial. [Tesis Título de Químico Farmacéutico]. Facultad de Farmacia y Bioquímica: Universidad Nacional Mayor de San Marcos. 2004

12. Foro: EnJambres migratorios del bosque: como obtener extracto Blando de Propoleo www.alimentosargentinos. gov.ar/programa calidad/ foros virtuales/apicola/mensajes/05 12 diciembre/varios 01.htm

13. Liébana UJ. Microbiología Oral. España: Editorial Interamericana. Mc Graw-Hill. 1995:258, 454, 455, 548

14. Evira EE, Villalobos DA, Andrade F. Estudio comparativo entre la eficacia del propóleos y la Clorhexidina en el manejo de las lesiones bucales, en pacientes pediátricos inmunodeprimidos. Rev Med Oral. 2001;3(3):109-114

Recibido :18-04-2007

Aceptado para publicación: 05-10-2007 